\title{
Development and internal validation of a prediction model of prostate cancer on initial transperineal template-guided prostate biopsy
}

\author{
Yuliang Chen ${ }^{1}$, Zhien Zhou' ${ }^{1}$ Yi Zhou ${ }^{1}$, Xingcheng Wu${ }^{1}$, Yu Xiao ${ }^{2}$, Zhigang Ji ${ }^{1}$, Hanzhong Li ${ }^{1}$ and Weigang Yan ${ }^{\text {* }}$
}

\begin{abstract}
Background: Due to the invasiveness of prostate biopsy, a prediction model of the individual risk of a positive biopsy result could be helpful to guide clinical decision-making. Most existing models are based on transrectal ultrasonography (TRUS)-guided biopsy. On the other hand, transperineal template-guided prostate biopsy (TTPB) has been reported to be more accurate in evaluating prostate cancer. The objective of this study is to develop a prediction model of the detection of high-grade prostate cancer (HGPC) on initial TTPB.
\end{abstract}

Result: A total of 1352 out of 3794 (35.6\%) patients were diagnosed with prostate cancer, 848 of whom had tumour with Grade Group 2-5. Age, PSA, PV, DRE and f/t PSA are independent predictors of HGPC with $p<0.001$. The model showed good discrimination ability (c-index 0.886) and calibration during internal validation and good clinical performance was observed through decision curve analysis. The external validation of CPCC-RC, an existing model, demonstrated that models based on TRUS-guided biopsy may underestimate the risk of HGPC in patients who underwent TTPB.

Conclusion: We established a prediction model which showed good discrimination ability and calibration in predicting the detection of HGPC by initial TTPB. This model can be used to aid clinical decision making for Chinese patients and other Asian populations with similar genomic backgrounds, after external validations are conducted to further confirm its clinical applicability.

Keywords: Transperineal template-guided prostate biopsy, High-grade prostate cancer, Prediction model, Nomogram

\section{Introduction}

According to GLOBOCAN data in 2018, the incidence and mortality of prostate cancer ranked second and fifth, respectively, among all cancers in men [1]. Prostate biopsy is essential for the diagnosis of prostate cancer.

\footnotetext{
*Correspondence: ywgpumch@sina.com

${ }^{1}$ The Department of Urology, Peking Union Medical College Hospital, Chinese Academy of Medical Sciences, No. 1 Shuaifuyuan, Dongcheng District, Beijing 100730, China

Full list of author information is available at the end of the article
}

Due to the invasive nature of biopsy, it would be very helpful if the individual risk of a positive biopsy result can be calculated through prediction models and guide clinical decision-making.

The incidence and prevalence of prostate cancer in Asian populations are significantly lower than in individuals of Caucasian and African descent [1, 2], suggesting ethnic differences in the occurrence of prostate cancer. At present, the most widely used and well-validated prediction models for prostate biopsy are the Prostate Cancer Prevention Trial Risk Calculator (PCPT-RC) and the 
European Randomized Study of Screening for Prostate Cancer Risk Calculator (ERSPC-RC) [3, 4]. However, the predicted risk of these models was reported to be overestimated by $20 \%$ in Chinese patients [5], which highlights the necessity of building a prediction model for Chinese patients, as well as Asian populations with similar genomic backgrounds.

Most of the existing models for the Chinese population, for example, the Chinese Prostate Cancer Risk Calculator (CPCC-RC), are based on transrectal ultrasonography (TRUS)-guided biopsy [6]. However, the transrectal approach has a higher probability of omitting tumours in the anterior prostate than does the transperineal approach [7]. On the other hand, transperineal template-guided prostate biopsy (ТTPB) is reported to be effective in detecting prostate cancer in patients with multiple negative transrectal biopsies, mainly due to improved sensitivity for anterior and apical tumours $[8$, 9]. Risk models based on TRUS-guided biopsy may not appropriately predict the risk of prostate cancer detected by transperineal biopsy.

The main objective of our study was to develop and internally validate a prediction model for the detection of high-grade prostate cancer (HGPC) by TTPB in biopsynaïve Chinese patients. In addition, we conducted an external validation of CPCC-RC, an existing prediction model based on TRUS-guided biopsy, to evaluate its performance in predicting TTPB results [10].

\section{Patients and methods}

\section{Study population and design}

We undertook a consecutive cohort study in prostate biopsy-naïve patients in our institution (Peking Union Medical College Hospital, a tertiary hospital in Beijing, China) between December 2003 and July 2019. The included patients met at least one of the following criteria: (1) prostate-specific antigen (PSA) $>4.0 \mathrm{ng} / \mathrm{mL}$; (2) abnormal findings on DRE; and (3) imaging results indicating the suspicion of prostate cancer. We excluded patients with PSA levels $>100.0 \mathrm{ng} / \mathrm{mL}$ or any history of prostate cancer, previous biopsy, endocrine treatment, or perineal surgery. A retrospective analysis of prospectively collected clinical data was performed.

The collection and use of all participant data and biological specimens in this study was ethically approved by the Ethics Committee of Peking Union Medical College Hospital, Chinese Academy of Medical Science. All the patients included in our study signed a written informed consent.

\section{Procedures}

As described in our previous study [11], TTPB was conducted in operation room with patients in lithotomy position. Local anesthesia was given to 3643 patients (96.0\%), with $10 \mathrm{ml}$ of $1 \%$ lidocaine injected intracutaneously and subcutaneously into the perineum, and another $10 \mathrm{~mL}$ onto the capsule to the right and left sides of the prostatic apex. The other 151 patients $(4.0 \%)$ received general anesthesia due to intolerance of pain or personal choice.

A biplanar TRUS probe (SONOLINE Adara SLC Ultrasound; Siemens, Erlangen, Germany) attached to a brachytherapy stepping unit (Computerized Medical System, St. Louis, MO, USA), and a standard $0.5-\mathrm{cm}$ brachytherapy template over the perineum were used to guide the transperineal biopsy. The length, width and height of the prostate were measured by ultrasound, and the prostate volume (PV) was calculated. One to four cores were taken by Bard biopsy gun (C.R. Bard, Covington, GA, USA) from each of the 11 regions [11], with more cores for larger prostates. The pathological assessment of biopsy specimens was conducted by two pathologists in our institution, one of whom has more than 10 years of experience in urological pathology.

\section{Pathological assessment}

All the biopsy cores were evaluated by at least 2 experienced urological pathologists in our institution according to the 2014 International Society of Urological Pathology (ISUP) modified Gleason system [12]. Overall GS was assigned after a comprehensive assessment of proportion of different Gleason patterns and distribution of each core. Discordant results from the 2 pathologists were resolved through discussion. Pathology slides and reports of cancerous specimens included in our study before 2015 were reviewed and updated by pathologists.

\section{Outcomes and predictors}

The outcome variable was the detection of HGPC, which in our study, was defined as prostate cancer with ISUP Grade Group $>1$ (Gleason score $>6$ ) because Grade Group 1 cancer is usually indolent and does not require aggressive treatments [13]. Referring to EAU guidelines [14], the two keynote risk calculators based on the Caucasian population $[3,4]$ and other prediction models of prostate biopsy [10, 15-17], we selected age, PSA level, PV, DRE, and free-to-total (f/t) PSA as potential predictors.

\section{Statistical analysis}

Statistical analysis was performed with $\mathrm{R}$ software (http://www.r-project.org/, version 4.0, Vienna, Austria). 
The mean \pm SD was used to describe data in a normal distribution, while the median and interquartile range were used for data in a skewed distribution. A multivariate logistic regression model was established with the detection of HGPC as the dependent variable. Age, PSA, PV, DRE, and $\mathrm{f} / \mathrm{t}$ PSA were included as potential predictors. Similar to other prediction models $[4,10]$, natural logarithm transformation was performed for PSA and PV to achieve better linearity with logit P. Independent variables that have a significant impact on the detection rate in univariate analysis were included in the final models after consideration of their clinical utility. A nomogram was developed based on significant predictors. Internal validation was performed with the concordance index (c-index) calculated and calibration curve depicted for the prediction model by bootstrapping with 1000 resamples. Decision curve analysis was conducted to evaluate the clinical performance of our models. A two-sided P value $<0.05$ denoted statistical significance.

Table 1 Characteristics of 3794 men in the development cohort

\begin{tabular}{ll}
\hline Parameter & $\begin{array}{l}\text { Development } \\
\text { cohort }(\mathbf{n}=\mathbf{3 7 9 4})\end{array}$ \\
\hline Age (years) & $68(61-74)$ \\
PSA (ng/ml)* & $10.00(6.80-15.78)$ \\
Abnormal DRE & $831(21.9 \%)$ \\
f/t PSA* & $0.143(0.098-0.200)$ \\
Prostate volume $(\mathrm{ml})^{*}$ & $45(35-60)$ \\
PC detected & $1352(35.6 \%)$ \\
ISUP grade group & \\
1 & 504 \\
2 & 279 \\
3 & 206 \\
4 & 151 \\
5 & 212 \\
HGPC detected & $848(22.4 \%)$ \\
In PC patients & $587(43.4 \%)$ \\
In HGPC patients & $456(53.8 \%)$ \\
\hline
\end{tabular}

${ }^{*}$ Data in skewed distribution described by median and interquartile range

\section{Results}

\section{Characteristics of participants}

A total of 3794 patients were enrolled consecutively from December 2003 to July 2019. The characteristics of all patients are shown in Table 1 . An average of 22.2 cores were taken from each patient with a median of 22 cores. 1352 out of 3794 (35.6\%) patients were diagnosed with prostate cancer, 848 of whom had tumours categorized as Grade group 2-5 according to ISUP consensus.

\section{Model development}

Age, $\log$ PSA, $\log$ PV, age, f/t PSA and DRE were significantly related to the detection of HGPC in univariate analysis and were also independent predictors of HGPC in multivariate logistic analysis (Table 2). To facilitate clinical use, we established a nomogram based on this prediction model (Fig. 1). The optimal cut-off of risk threshold which maximized Youden index (sensitivity + specificity -1 ) was calculated to be 0.244 (sensitivity $=0.839$, specificity $=0.748)$.

\section{Internal validation}

The apparent C-index of our model is 0.866. Using 1000-resample bootstrapping, the optimism of our model is calculated to be 0.002 , and the bias-corrected C-index to be 0.865 , demonstrating good discrimination ability. The calibration curve, also depicted by bootstrapping, is shown in Fig. 2a with excellent calibration between predictions and observations (bias-corrected slope $=0.994$, bias-corrected intercept $=-0.004)$.

\section{External validation of an existing model}

Using our TTPB data, we conducted external validation of CPCC-RC [10], which was an existing prediction model for the Chinese population based on a transrectal approach. The C-index was 0.826 when predicting HGPC using the CPCC-RC. The upwardly curved calibration curve in Fig. 2b suggests that the CPCC-RC underestimates the risk of HGPC in patients who undergo TTPB.

Table 2 Model predicting the detection of high-grade prostate cancer on initial transperineal template-guided prostate biopsy

\begin{tabular}{|c|c|c|c|c|c|c|}
\hline \multirow[t]{2}{*}{ Predictor } & \multicolumn{3}{|l|}{ Univariate analysis } & \multicolumn{3}{|l|}{ Multivariable model } \\
\hline & OR(95\% Cl) & $\beta$ & $\mathbf{P}$ & Adjusted OR (95\% Cl) & $\beta$ & $P$ \\
\hline Age & $1.0691(1.0589-1.0794)$ & 0.0668 & $<0.001$ & $1.0686(1.0563-1.0809)$ & 0.0663 & $<0.001$ \\
\hline DRE & $7.9510(6.6878-9.4529)$ & 2.0733 & $<0.001$ & $3.9141(3.1915-4.8002)$ & 1.3646 & $<0.001$ \\
\hline $\log P S A$ & $3.7242(3.3035-4.1985)$ & 1.3149 & $<0.001$ & $2.8486(2.4842-3.2665)$ & 1.0468 & $<0.001$ \\
\hline $\log P V$ & $0.2128(0.1731-0.2618)$ & -1.5470 & $<0.001$ & $0.1728(0.1323-0.2257)$ & -1.7556 & $<0.001$ \\
\hline f/t PSA & $0.0006(0.0002-0.0023)$ & -7.2973 & $<0.001$ & $0.0305(0.0073-0.1276)$ & -3.4910 & $<0.001$ \\
\hline Intercept & - & - & - & - & -1.7803 & - \\
\hline
\end{tabular}




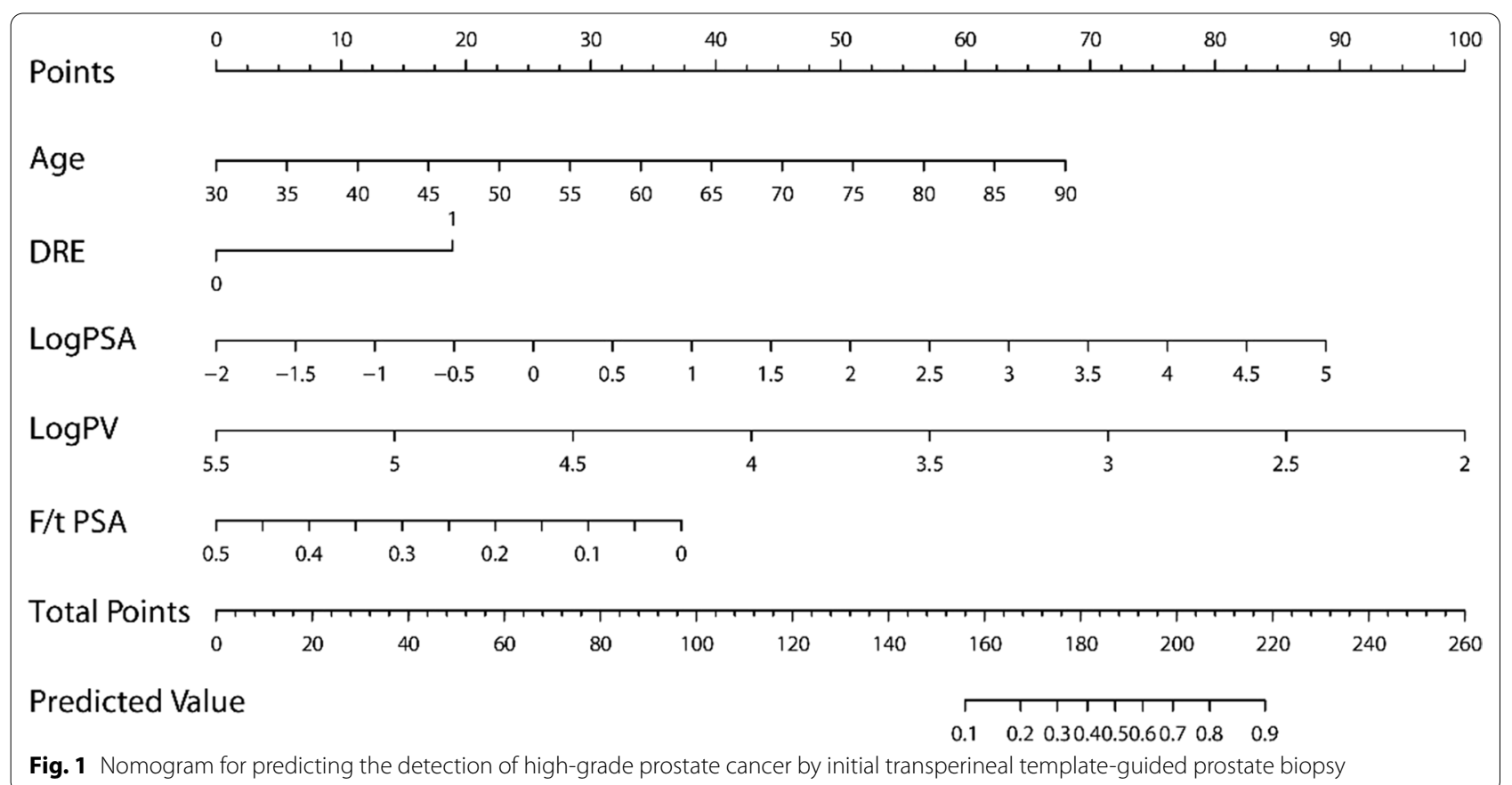
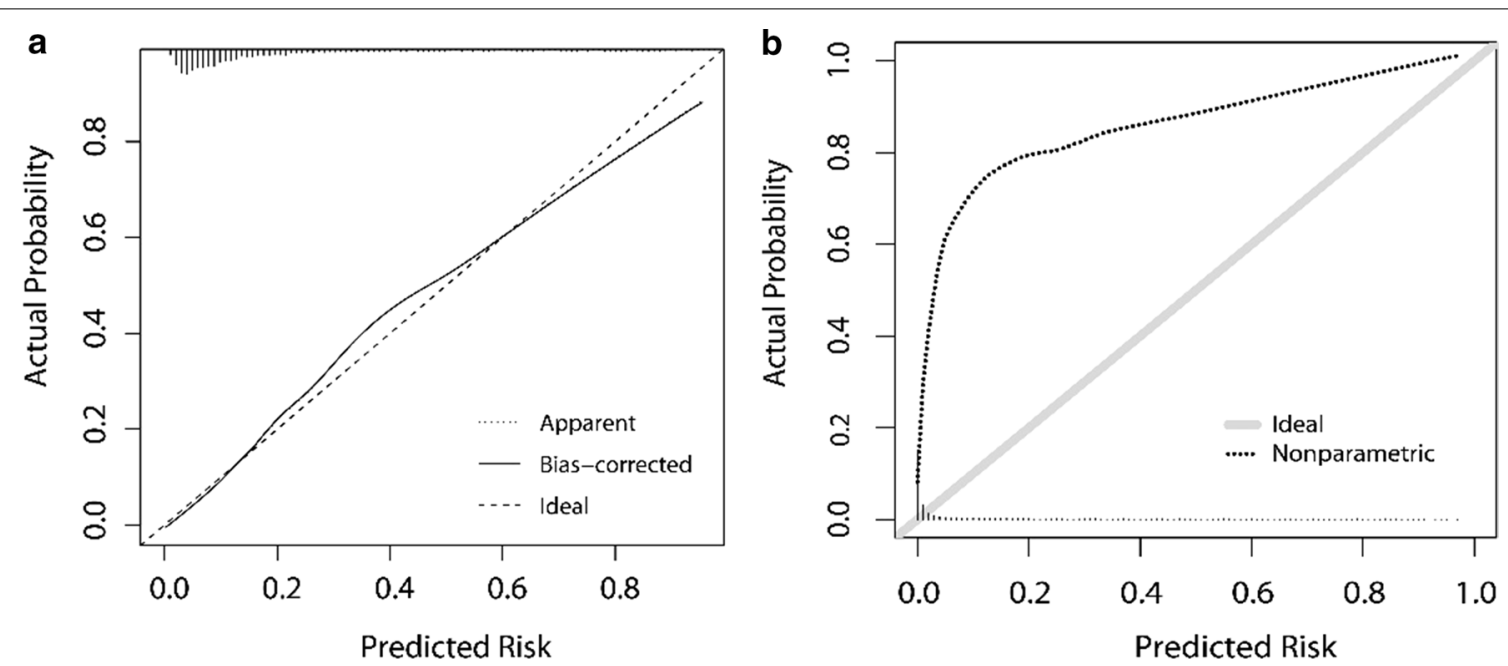

Fig. 2 Calibration curves for the prediction models. a Our model in internal validation by 1000-resample bootstrapping. b The CPCC-RC in external validation using TTPB data

\section{Decision curve analysis}

We performed decision curve analysis for our model. In Fig. 3, the net benefit of conducting biopsy for all or none of the patients, which are two extreme situations, is represented by the grey line and the horizonal black line, respectively. In a wide range of risk thresholds, our model outperformed the two extreme strategies with a much higher net benefit. For example, if we use 0.3 as a risk threshold to determine whether TTPB is required according to our model, after weighing the benefit and cost, there is a net benefit for 11 out of every 100 people.

\section{Discussion}

Although there have been prediction models for the detection of HGPC in Chinese population [10, 16-18], our study is the first to be based on TTPB to the best of our knowledge. Compared with the transrectal approach, transperineal biopsy is less likely to cause rectal bleeding $(\mathrm{RR}=0.02,95 \% \mathrm{CI} 0.01-0.06)$ and fever $(\mathrm{RR}=0.26,95 \%$ 


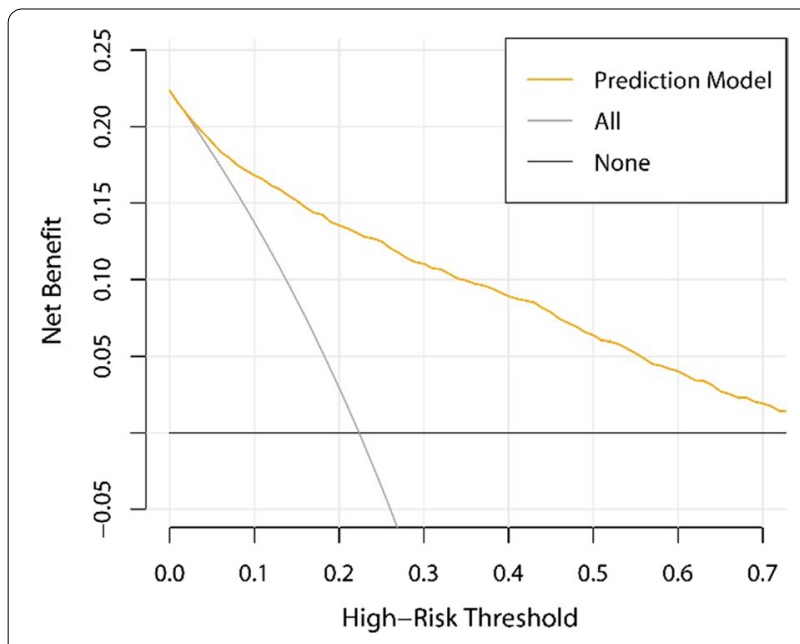

Fig. 3 Decision curve analysis

CI 0.14-0.28) according to a meta-analysis by Xiang et al. [7]. A recent research based on UK National Health Service data demonstrated that from 2017 to 2019, the sepsis rate of transperineal biopsy was significantly lower than that of transrectal approach $(0.42 \%$ vs. $1.12 \%, \mathrm{p}<0.001)$ [19]. On the other hand, transperineal biopsy was reported to be associated with severer pain (VAS score: 4.0 vs. 2.0$)$, higher rate of additional anesthesia $(15.0 \%$ vs. $1.2 \%)$ and extended operation time $(17.51 \pm 3.33 \mathrm{~min}$ vs. $14.73 \pm 3.25 \mathrm{~min})$ in comparison with transrectal approach [20].

While no significant difference was observed between the positive rate of transrectal and transperineal biopsy according to a meta-analysis [7], there has been evidence that the transperineal approach exceeds transrectal biopsy in terms of accurate diagnosis and risk assessment of prostate cancer [21-23]. A multi-centre autopsy study revealed that the proportion of anterior tumours did not significantly differ from that of posterior ones [24]. A higher detection rate of TTPB than of TRUS-guided biopsy was observed for anterior prostate cancer, possibly due to the difference in sites where the biopsy cores are taken [23]. When TTPB was given to active surveillance patients within 12 months of diagnosis by TRUS biopsy, histopathological upgrading was observed in $38.8 \%(83 / 208)$ of them by Voss et al. [21]. TTPB also demonstrated better concordance with radical prostatectomy pathology than did TRUS biopsy [22].

Although these two approaches were not directly compared in our study, we conducted external validation of the CPCC-RC. With our data collected from a similar population, the predicted risk of HGPC is underestimated by the CPCC-RC for a wide range of risk thresholds, suggesting that TTPB might be more sensitive in detecting HGPC. Of course, we must take into account the presence of different operators and pathologists between our cohort and the CPCC-RC, which may have influenced biopsy results.

MRI-targeted biopsy was reported to surpasses systematic biopsy in detecting high-grade prostate cancer [25] but still omits approximately $10 \%$ of clinically significant prostate cancer in patients with MRI-visible lesions [26]. As a result, systematic biopsies are typically suggested in addition to the MRI targeted biopsies. Meanwhile, due to the requirement of equipment and the high cost of MRI, patients with poor medical resource or poor financial conditions may not have access to MRI examination at the first visit or simply not be willing to receive one. For them, available clinical data are age, PSA level, prostate volume, and DRE result. Our models can be used during such biopsy counselling, when benefit and harm can be weighed by doctors and patients through the predicted probability of a positive biopsy result. With data from the developing cohort, we estimated the risk threshold for recommending TTPB to be $24.4 \%$, which is in concordance with the empirical threshold of $25 \%$ [4]. This threshold can also be personalized during consultation. For patients with low predicted risk, observation might be a choice. Average risk patients might benefit from MRI for further risk assessment and high-risk patients might be recommended for MRI and biopsies.

Our study does have some limitations. First, some significant small tumours may have been missed in TTPB. In our study, this rate is not known, since it is not feasible to compare TTPB result with post prostatectomy or transperineal saturation biopsy results for each patient. Hence, some patients might need some follow up or further investigations even if the predicted risk is low. Second, some novel clinical indicators with better effects, such as Prostate Health Index, 4-Kallikrein Panel Score and Genomic Score [27], were not included in our study. Considering that such indicators are difficult to obtain in areas with general medical conditions, those included in our study are closer to practical applications. Finally, participants of our research were enrolled from a single centre, and further external validation is required to confirm the clinical applicability of our model.

\section{Conclusion}

We established and internally validated a prediction model for the detection of HGPC on initial TTPB in a Chinese population. Good clinical performance was indicated by decision curve analysis. External validations are required to further confirm the efficacy of our model.

\section{Acknowledgements}

Not applicable. 


\section{Authors' contributions}

Y Chen: project development, data analysis, manuscript writing; Z Zhou: project development, data collection, manuscript editing; $Y$ Zhou: data collection, manuscript editing; $X$ Wu: data collection and analysis; $Y$ Xiao: data collection and analysis, Z Ji: data collection, manuscript editing; H Li: data collection, manuscript editing; W Yan: project development, data collection, manuscript editing. All authors read and approved the final manuscript.

\section{Funding}

This research did not receive any specific grant from funding agencies in the public, commercial, or not-for-profit sectors.

\section{Availability of data and materials}

The datasets used and analysed during the current study are available from the corresponding author on reasonable request.

\section{Declarations}

\section{Ethics approval and consent to participate}

This study was performed in line with the principles of the Declaration of Helsinki. Approval was granted by the Ethics Committee of Peking Union Medical College Hospital, Chinese Academy of Medical Science. Informed consent was obtained from all individual participants included in the study.

\section{Consent for publication}

Informed consent for publication was obtained from all individual participants included in the study.

\section{Competing interests}

Weigang Yan certifies that there is no conflict of interest related to this article for all authors.

\section{Author details}

${ }^{1}$ The Department of Urology, Peking Union Medical College Hospital, Chinese Academy of Medical Sciences, No. 1 Shuaifuyuan, Dongcheng District, Beijing 100730, China. ${ }^{2}$ The Department of Pathology, Peking Union Medical College Hospital, Chinese Academy of Medical Sciences, No. 1 Shuaifuyuan, Dongcheng District, Beijing 100730, China.

Received: 3 November 2020 Accepted: 12 April 2021 Published online: 23 April 2021

\section{References}

1. Bray F, Ferlay J, Soerjomataram I, Siegel RL, Torre LA, Jemal A. Global cancer statistics 2018: GLOBOCAN estimates of incidence and mortality worldwide for 36 cancers in 185 countries. CA Cancer J Clin. 2018;68(6):394-424.

2. RebbeckTR, Haas GP. Temporal trends and racial disparities in global prostate cancer prevalence. Can J Urol. 2014;21(5):7496-506.

3. Roobol MJ, Steyerberg EW, Kranse R, Wolters T, van den Bergh RCN, Bangma $\mathrm{CH}$, et al. A risk-based strategy improves prostate-specific antigen-driven detection of prostate cancer. Eur Urol. 2010;57(1):79-85.

4. Thompson IM, Ankerst DP, Chi C, Goodman PJ, Tangen CM, Lucia MS, et al. Assessing prostate cancer risk: results from the Prostate Cancer Prevention Trial. J Natl Cancer Inst. 2006;98(8):529-34.

5. Zhu Y, Wang JY, Shen YJ, Dai B, Ma CG, Xiao WJ, et al. External validation of the Prostate Cancer Prevention Trial and the European Randomized Study of Screening for Prostate Cancer risk calculators in a Chinese cohort. Asian J Androl. 2012;14(5):738-44

6. He B-M, Chen R, Sun T-Q, Yang Y, Zhang C-L, Ren S-C, et al. Prostate cancer risk prediction models in Eastern Asian populations: current status, racial difference, and future directions. Asian J Androl. 2020;22(2):158-61.

7. Xiang J, Yan H, Li J, Wang X, Chen H, Zheng X. Transperineal versus transrectal prostate biopsy in the diagnosis of prostate cancer: a systematic review and meta-analysis. World J Surg Oncol. 2019;17(1):31.

8. Gershman B, Zietman AL, Feldman AS, McDougal WS. Transperineal template-guided prostate biopsy for patients with persistently elevated PSA and multiple prior negative biopsies. Urol Oncol. 2013;31(7):1093-7.

9. Pal RP, Elmussareh M, Chanawani M, Khan MA. The role of a standardized 36 core template-assisted transperineal prostate biopsy technique in patients with previously negative transrectal ultrasonographyguided prostate biopsies. BJU Int. 2012;109(3):367-71.

10. Chen R, Xie L, Xue W, Ye Z, Ma L, Gao X, et al. Development and external multicenter validation of Chinese Prostate Cancer Consortium prostate cancer risk calculator for initial prostate biopsy. Urol Oncol. 2016;34(9):416.e1-e7.

11. Mai Z, Yan W, Zhou Y, Zhou Z, Chen J, Xiao Y, et al. Transperineal template-guided prostate biopsy: 10 years of experience. BJU Int 2016:117(3):424-9.

12. Epstein JI, Egevad L, Amin MB, Delahunt B, Srigley JR, Humphrey PA. The 2014 International Society of Urological Pathology (ISUP) consensus conference on gleason grading of prostatic carcinoma: definition of grading patterns and proposal for a new grading system. Am J Surg Pathol. 2016:40(2):244-52.

13. Epstein Jl, Amin MB, Reuter VE, Humphrey PA. Contemporary Gleason Grading of Prostatic Carcinoma: an update with discussion on practical issues to implement the 2014 International Society of Urological Pathology (ISUP) consensus conference on Gleason Grading of Prostatic Carcinoma. Am J Surg Pathol. 2017;41(4):e1-e7.

14. Mottet NPC, den Bergh RCN, Briers E, De Santis M, Fanti S, Gillessen S, Grummet AM, Henry AM, Lam TB, Mason HG, van der Kwast HG, van der Poel HG, Rouvière $O$, Schoots $D$, Tilki $D$, Wiegel T. EAU-EANMESTRO-ESUR-SIOG Guidelines on Prostate Cancer: Uropean Association of Urology; 2020. https://uroweb.org/guideline/prostate-cancer/.

15. Wu Y-S, Zhang N, Liu S-H, Xu J-F, Tong S-J, Cai Y-H, et al. The Huashan risk calculators performed better in prediction of prostate cancer in Chinese population: a training study followed by a validation study. Asian J Androl. 2016;18(6):925-9.

16. Huang Y, Cheng G, Liu B, Shao P, Qin C, Li J, et al. A prostate biopsy strategy based on a new clinical nomogram reduces the number of biopsy cores required in high-risk patients. BMC Urol. 2014;14:8.

17. Tang P, Chen H, Uhlman M, Lin Y-R, Deng X-R, Wang B, et al. A nomogram based on age, prostate-specific antigen level, prostate volume and digital rectal examination for predicting risk of prostate cancer. Asian J Androl. 2013;15(1):129-33.

18. Kuo SC, Hung SH, Wang HY, Chien CC, Lu CL, Lin HJ, et al. Chinese nomogram to predict probability of positive initial prostate biopsy: a study in Taiwan region. Asian J Androl. 2013;15(6):780-4.

19. Tamhankar AS, El-Taji O, Vasdev N, Foley C, Popert R, Adshead J. The clinical and financial implications of a decade of prostate biopsies in the NHS: analysis of Hospital Episode Statistics data 2008-2019. BJU Int. 2020;126(1):133-41.

20. Guo L-H, Wu R, Xu H-X, Xu J-M, Wu J, Wang S, et al. Comparison between ultrasound guided transperineal and transrectal prostate biopsy: a prospective, randomized, and controlled trial. Sci Rep. 2015;5:16089.

21. Voss J, Pal R, Ahmed S, Hannah M, Jaulim A, Walton T. Utility of early transperineal template-guided prostate biopsy for risk stratification in men undergoing active surveillance for prostate cancer. BJU Int. 2018;121(6):863-70.

22. Marra G, Eldred-Evans D, Challacombe B, Van Hemelrijck M, Polson A, Pomplun S, et al. Pathological concordance between prostate biopsies and radical prostatectomy using transperineal sector mapping biopsies: validation and comparison with transrectal biopsies. Urol Int. 2017;99(2):168-76.

23. Hossack T, Patel MI, Huo A, Brenner P, Yuen C, Spernat D, et al. Location and pathological characteristics of cancers in radical prostatectomy specimens identified by transperineal biopsy compared to transrectal biopsy. J Urol. 2012;188(3):781-5.

24. Breslow N, Chan CW, Dhom G, Drury RA, Franks LM, Gellei B, et al. Latent carcinoma of prostate at autopsy in seven areas the International Agency for Research on Cancer, Lyons, France. Int J Cancer. 1977;20(5):680-8.

25. Siddiqui MM, Rais-Bahrami S, Turkbey B, George AK, Rothwax J, Shakir $\mathrm{N}$, et al. Comparison of MR/ultrasound fusion-guided biopsy with ultrasound-guided biopsy for the diagnosis of prostate cancer. JAMA. 2015;313(4):390-7. 
26. Ahdoot M, Wilbur AR, Reese SE, Lebastchi AH, Mehralivand S, Gomella PT, et al. MRI-targeted, systematic, and combined biopsy for prostate cancer diagnosis. N Engl J Med. 2020;382(10):917-28.

27. Srivastava S, Koay EJ, Borowsky AD, De Marzo AM, Ghosh S, Wagner PD, et al. Cancer overdiagnosis: a biological challenge and clinical dilemma. Nat Rev Cancer. 2019;19(6):349-58.

\section{Publisher's Note}

Springer Nature remains neutral with regard to jurisdictional claims in published maps and institutional affiliations.
Ready to submit your research? Choose BMC and benefit from:

- fast, convenient online submission

- thorough peer review by experienced researchers in your field

- rapid publication on acceptance

- support for research data, including large and complex data types

- gold Open Access which fosters wider collaboration and increased citations

- maximum visibility for your research: over $100 \mathrm{M}$ website views per year

At BMC, research is always in progress.

Learn more biomedcentral.com/submissions 